\section{Avaliação do déficit bilateral em contrações isométricas dos extensores de joelhos}

\section{Evaluation of bilateral deficit in isometric contractions of the knee extensors}

\author{
Ronei Silveira Pinto ${ }^{1}$ \\ Cíntia Ehlers Botton ${ }^{1}$ \\ Bruno Tomasi Kuckartz ${ }^{1}$ \\ Cláudia Silveira Lima \\ Antônio Carlos de Moraes ${ }^{2}$ \\ Martim Bottaro 3
}

Resumo - O treinamento de força tem sido referido como uma importante ferramenta para o incremento e a manutenção da saúde. Os exercícios que compõem um programa de treino podem ser executados em condição unilateral ou bilateral. Alguns estudos têm apresentado valores mais reduzidos de produção de força e sinal eletromiográfico em condições bilaterais do que o somatório desses valores em condições unilaterais. Essa diferença na produção de força, bem como no sinal eletromiográfico é denominada déficit bilateral. O objetivo do presente estudo foi avaliar a produção de força e a atividade eletromiográfica dos músculos reto da coxa e vasto lateral no exercício de extensão isométrica de joelhos, nas condições unilateral e bilateral. A amostra foi constituída de 10 indivíduos do sexo masculino, com idades entre 18 e 30 anos, não praticantes de treinamento de força. Foram registrados os valores de força e atividade elétrica com a utilização de uma célula de carga e eletrodos de superfície, respectivamente, no teste isométrico de extensão de joelhos $\left(120^{\circ}\right)$. Os resultados apontaram valores maiores $(\mathrm{p}<0,05)$ para a produção de força e sinal eletromiográfico na condição unilateral do exercício, reportando um déficit bilateral tanto na força como na atividade eletromiográfica. Estes resultados sugerem que o treino unilateral promove maior estímulo muscular, constituindo-se como uma importante estratégia a ser utilizada na prescrição dos exercícios de força.

Palavras-chave: Déficit bilateral; Eletromiografia; Força muscular.

Abstract - Strength training has been reported as an important tool for enhancing and maintaining health. Resistance exercises can be executed unilaterally or bilaterally. Some studies have reported lower strength and electromyography (EMG) signal values in bilateral conditions than the sum of these values in unilateral conditions. This difference in strength and EMG is called the bilateral deficit. The aim of this study was to evaluate the strength and EMG activity of the rectus femoris and vastus lateralis during unilateral and bilateral isometric knee extension. The sample consisted of 10 non-resistance trained males aged 18 to 30 years. Strength and EMG were recorded through use of a load cell and surface electrodes, respectively. A $120^{\circ}$ knee extension was used for the isometric knee extension test. The results showed significantly higher values for strength and EMG signal $(p<0.05)$ in unilateral conditions, as well as bilateral deficit in both strength and EMG signal. These results suggest that unilateral training promotes greater muscle stimulation, and can thus represent an important strategy to be used in the prescription of strength training exercises. Key words: Bilateral deficit; Electromyography; Muscle strength.
1 Universidade Federal do Rio Grande do Sul. Escola de Educação Física. Porto Alegre, RS. Brasil.

2 Universidade Estadual de Campinas. Faculdade de Educação Física. Campinas, SP.Brasil.

3 Universidade de Brasília. Faculdade de Educação Física. Brasília, DF, Brasil.

Recebido em 08/07/11 Revisado em 20/08/11 Aprovado em 07/10/11 


\section{INTRODUÇÃO}

O treinamento de força tornou-se um dos tipos de exercício físico mais popular para melhora da aptidão física e, nesta perspectiva, tem sido utilizado por atletas e não atletas ${ }^{1}$. A escolha dos exercícios de força constitui-se numa das principais variáveis agudas na prescrição do treinamento ${ }^{2}$. Quanto à lateralidade, os exercícios que compõem as sessões de treino podem ser executados na condição bilateral (BL), quando realizados com os membros homólogos simultaneamente, ou na condição unilateral (UL), quando realizados com apenas um dos membros por vez ${ }^{3}$.

Alguns estudos ${ }^{3-7}$ demonstram diferença significativa entre a produção de força máxima durante ações bilaterais, quando comparadas às ações unilaterais, nas quais o somatório da produção de força dos membros contralaterais é superior à força produzida bilateralmente. Esta diferença tem sido denominada déficit bilateral (DB), e pode ser avaliada pelo índice bilateral (IB). O IB pode ser calculado a partir da força produzida bilateralmente em uma contração isométrica voluntária máxima (CIVM BL) comparada ao somatório das forças produzidas unilateralmente nas mesmas condições (membro direito - CIVM ULD + membro esquerdo - CIVM ULE), e pode ser calculado a partir de equações específicas, como a proposta por Howard e Enoka ${ }^{8}$ IB = [100 x (CIVM BL / (CIVM ULD+CIVM ULE) $]$-100. Valores negativos (inferiores a zero) relativos ao IB indicam a presença de DB, enquanto valores positivos (superiores a zero) indicam o que é denominado de facilitação bilateral. Da mesma forma que em condições isométricas, o DB pode ser também calculado em condições dinâmicas, tendo sido esta forma menos investigada até o momento ${ }^{4,9}$. O IB também pode ser calculado com a utilização da eletromiografia (EMG), obedecendo ao mesmo princípio acima descrito e, quando ocorrido, explicita menores níveis de ativação muscular na condição $B L^{6,7,9}$.

Os mecanismos mais referidos na literatura para explicar o fenômeno DB são relacionados à ativação diferenciada dos hemisférios cerebrais, nas duas condições (UL e BL) e ao menor nível de ativação de fibras tipo II (de contração rápida) na condição BL. Segundo Owings e Grabiner ${ }^{3}$, a redução do desempenho muscular durante movimentos bilaterais (membros sincronizados) parece estar associada ao menor nível de ativação dos hemisférios cerebrais observado nestas condições. Quando os dois hemisférios cerebrais são simultaneamente ativados, o que ocorre durante a execução dos exercícios bilaterais, parece haver uma inibição da ativação cerebral total, resultando na diminuição da estimulação de unidades motoras e, consequentemente, menor produção de força de cada membro contralateral neste modo de execução 5 . Outro mecanismo que parece também explicar o DB é o menor nível de ativação

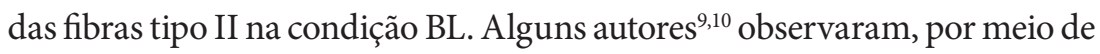
protocolos de fadiga ou de diferentes velocidades, que o DB estava associado ao reduzido recrutamento de fibras tipo II durante contrações bilaterais.

No entanto, as divergências encontradas na literatura quanto à ocorrência de diferenças na produção de força e nível de ativação muscular nos 
exercícios unilaterais e bilaterais (i.e. déficit bilateral ou facilitação bilateral) quando executados de forma isométrica máxima, fragilizam a especulação de que os exercícios unilaterais incrementam, de forma crônica, mais a força e a hipertrofia muscular do que os exercícios bilaterais. Alguns autores reportaram a presença do déficit em seus estudos ${ }^{4,5,8,10,11}$, enquanto outros não o observaram ${ }^{12,13-15}$, o que torna o tema ainda inconclusivo. Assim, o objetivo do presente estudo foi avaliar e comparar a produção de força máxima e o nível de ativação muscular no exercício de extensão isométrica do joelho realizado de forma UL e BL.

\section{PROCEDIMENTOS METODOLÓGICOS}

\section{Sujeitos}

Tendo-se como referência o estudo de Kuruganti e Murphy ${ }^{6}$, em que as condições experimentais assemelharam-se às do presente estudo, o tamanho da amostra foi calculado a partir da utilização do programa PEPI, versão 4.0 , adotando-se um nível de significância de 0,05 e um poder de $80 \%$. O referido cálculo demonstrou a necessidade de um número mínimo de nove sujeitos. Desta forma, 10 indivíduos do sexo masculino com idade entre 18 e 30 anos (74,7 $\pm 9,9 \mathrm{~kg} ; 1,80 \pm 0,08 \mathrm{~m}$ ), não praticantes do treino de força há pelo menos seis meses anteriores ao início do estudo, fizeram parte da amostra. Esta investigação foi aprovada pelo Comitê de Ética em Pesquisa institucional, conforme a Resolução 196/96 do Conselho Nacional de Saúde, pelo número 2008012.

\section{Avaliação da Força Isométrica}

Cada indivíduo compareceu três vezes ao laboratório, sendo um dia para familiarização com os protocolos de teste, um dia para realização dos testes na condição UL (ULD/ULE) e outro dia para realização do teste na condição $\mathrm{BL}$, com intervalo de 48 horas entre os dias. O protocolo para avaliação da força isométrica UL e BL consistiu de três contrações isométricas voluntárias máximas (CIVMs) de extensão de joelhos para cada uma das condições: BL (membros homólogos simultaneamente), ULD (apenas o membro direito) e ULE (apenas o membro esquerdo). Cada CIVM foi realizada durante cinco segundos, com intervalo de repouso de cinco minutos entre as tentativas ${ }^{20}$. Os sujeitos receberam estímulo verbal padronizado durante a coleta de dados.

No primeiro dia, os sujeitos foram informados sobre os objetivos e detalhes metodológicos do estudo, e assinaram o Termo de Consentimento Informado. Posteriormente, participaram de uma sessão de familiarização com os protocolos de avaliação nas condições de execução UL e BL. A familiarização foi semelhante ao protocolo de avaliação, consistindo de duas CIVMs para cada condição, com duração de cinco segundos e intervalo de um minuto. Os sujeitos receberam instrução para manter a força máxima durante todo período da contração e também foram familiarizados com o estímulo verbal durante os testes. 
No segundo e terceiro dia, foi realizado o protocolo de avaliação da força isométrica máxima (em N) em uma das condições de execução, UL (ULD e ULE) ou BL, conforme randomização. No dia em que foi realizado o teste UL, a ordem, direito/esquerdo, foi estabelecida por sorteio. Antes de iniciar o teste, os indivíduos foram submetidos a um prévio aquecimento no equipamento de extensão de joelhos, que consistiu em 15 repetições de extensão de joelhos com uma carga (leve), equivalente a 20\% da massa corporal. Após, foram realizadas as tentativas para a coleta das CIVMs e, simultaneamente, do sinal eletromiográfico (EMG).

Para a coleta de dados das CIVMs nas condições UL e BL, foi utilizado um equipamento de extensão de joelhos (Taurus, Porto Alegre, RS, Brasil), equipado com uma célula de carga (Miotec, Porto Alegre, RS, Brasil), com capacidade de $2000 \mathrm{~N}$, para a aquisição da curva de força-tempo. Os indivíduos foram posicionados no equipamento de extensão dos joelhos e o encosto da cadeira foi ajustado para cada indivíduo de forma que a articulação do quadril ficasse em um ângulo de $110^{\circ}$ e de modo que as CIVMs de extensão de joelhos fossem realizadas no ângulo de $120^{\circ}$ de extensão de joelho $\left(180^{\circ}=\right.$ extensão completa). Um goniômetro em acrílico (Marca Pro-Fisiomed, Porto Alegre, RS, Brasil) com resolução de um grau foi utilizado para o posicionamento do joelho no ângulo desejado com os sujeitos em contração. A fixação dos sujeitos ao equipamento foi realizada com faixas de velcro posicionadas na altura do tórax e os braços foram mantidos lateralmente, com as mãos seguras nos locais específicos de empunhadura do equipamento.

\section{Avaliação do sinal Eletromiográfico (EMG)}

A coleta do sinal EMG ocorreu concomitante aos testes de força (CIVMs). Assim, os sinais EMG e de força estavam sincronizados. Foram utilizados eletrodos de superfície bipolares (Meditrace. Porto Alegre, RS, Brasil) posicionados no ventre dos músculos reto da coxa (RC) e vasto lateral (VL), de ambos os membros. De acordo com Leis e Trapani ${ }^{16}$, o posicionamento dos eletrodos no músculo RC foi feito no ponto médio entre a espinha ilíaca ântero-superior (origem proximal) e a patela (origem distal) na parte anterior da coxa. No músculo VL os eletrodos foram posicionados de 8 a 10 centímetros acima da patela, localizado na região ântero-lateral da coxa. Antes da colocação dos eletrodos, foi realizada a tricotomia e abrasão com algodão e álcool para a retirada de gordura e células mortas da pele, a fim de reduzir a impedância.

A distância intereletrodos foi estabelecida em $20 \mathrm{~mm}$. O nível de resistência entre os eletrodos foi medido e controlado antes de cada sessão com um multímetro digital, devendo manter-se abaixo de $3000 \mathrm{Ohms}^{17}$. O eletrodo de referência foi posicionado na clavícula. Para minimizar o erro de posicionamento dos eletrodos entre as sessões, foram feitos mapas de avaliação, de acordo com a proposição de Narici et al. ${ }^{17}$.

Para a coleta dos dados de força (CIVMs) e EMG, foram utilizados dois eletromiógrafos Miotool 400 (Miotec. Porto Alegre, RS, Brasil). Cada 
equipamento é composto por um sistema de quatro canais, com frequência de amostragem de $2000 \mathrm{~Hz}$ por canal e ganho de 100. Os dados coletados foram transmitidos a um microcomputador portátil, contendo o software Miograph, versão 20.0 (Miotec. Porto Alegre, RS, Brasil), que possibilitou a visualização das avaliações, em tempo real.

\section{Análise dos dados}

O tratamento e a análise dos dados de produção de força e sinal EMG foram realizados no Sistema Aquisição de Dados 32 (SAD32, versão 2.61. Porto Alegre, RS, Brasil). Para a produção de força, o valor médio representativo de cada CIVM foi obtido através de um recorte de aproximadamente um segundo, realizado visualmente por um único avaliador, no platô da curva de força, em que esta era máxima e apresentava-se constante. Inicialmente, o sinal referente à curva de força foi filtrado, utilizando-se um filtro digital do tipo Passa-baixa Butterworth, $4^{\mathrm{a}}$ ordem, com frequência de corte de 4 Hz. Posteriormente, foi realizado o recorte e então, o maior valor registrado entre as três CIVMs foi considerado, para cada uma das condições avaliadas (ULD, ULE e BL), e utilizado para posterior comparação entre a soma dos valores de produção de força dos membros (direito + esquerdo) na condição UL ( $\Sigma$ UL) e os valores de produção de força BL.

Quanto ao sinal EMG, foi analisado o sinal da CIVM com maior valor de força. Primeiramente, foi realizada a filtragem digital do sinal utilizando-se um filtro do tipo Passa-alta Butterworth, $5^{\mathrm{a}}$ ordem, com frequência de corte de $20 \mathrm{~Hz}$. A seguir, foi calculado o valor root mean square (RMS) dos músculos RC e VL, no mesmo período de tempo ( 1s) em que os valores de força foram calculados, sendo o sinal EMG de cada músculo recortado no platô da curva de força. Os valores RMS relativos aos músculos RC e VL foram somados, para posterior análise.

\section{Índice bilateral}

Os maiores valores entre as três CIVMs para cada condição (ULD, ULE e BL) foram utilizados para avaliar o IB de produção de força máxima dos sujeitos, sendo inseridos na equação proposta por Howard e Enoka ${ }^{8}: \mathrm{IB}_{f}(\%)$ $=100 x[C I V M B L /(C I V M U L D+C I V M U L E)]-100$. Valores de IB $_{\mathrm{f}}(\%)$ menores que zero indicam presença de $\mathrm{DB}$, enquanto valores positivos indicam presença de facilitação bilateral.

Para avaliar o IB da ativação muscular, os valores do sinal EMG em RMS da soma dos músculos (VL+RC) de cada membro foram inseridos na equação conforme Koh et al..$^{10}$ :

$$
\mathrm{B}_{\text {emg }}(\%)=100 x\left[B L_{\text {esquerdo }}+\mathrm{BL}_{\text {direito }} / \mathrm{UL}_{\text {esquerdo }}+\mathrm{UL}_{\text {direito }}\right]-100
$$

Em que, $\mathrm{BL}_{\text {esquerdo }}=$ soma dos valores RMS da atividade EMG dos músculos VL e $\mathrm{RC}$ do membro inferior esquerdo na condição bilateral; $B L=$ soma dos valores RMS da atividade EMG dos músculos VL e RC do membro inferior direito na condição bilateral; $U L_{\text {esquerdo }}$ : soma dos valores RMS da atividade EMG dos músculos VL e RC do membro inferior esquerdo na condição unilateral; $U_{L_{\text {direito }}}$ : soma dos valores RMS da atividade EMG dos músculos VL e RC do membro inferior direito na condição unilateral. 
Desta forma, o IB é referente à comparação da soma da ativação muscular (VL+RC) dos membros esquerdo e direito nas condições UL e BL. Valores de $\mathrm{IB}_{\text {emg }}(\%)$ menores que zero indicam que os valores do sinal EMG UL foram maiores que os valores do sinal EMG BL. Já valores $\mathrm{IB}_{\text {emg }}(\%)$ maiores que zero indicam que os valores do sinal EMG BL são maiores que os valores na condição $\mathrm{BL}$.

\section{Análise Estatística}

Para a análise estatística dos dados, foi utilizada a estatística descritiva (média \pm desvio-padrão). A comparação dos valores de produção de força máxima nas condições UL e BL foi realizada por meio de um teste $t$ para amostras pareadas. Quanto ao IB, um teste $t$ para amostra única foi utilizado para indicar se $\mathrm{o} \mathrm{IB}_{\mathrm{f}} \mathrm{e} \mathrm{IB}_{\mathrm{emg}}$ foram diferentes de zero. O nível de significância dos testes foi considerado de $\mathrm{p}<0,05$. Os cálculos estatísticos foram realizados no programa SPSS, versão 18.0 (SPSS Inc. Chicago, IL, EUA).

\section{RESULTADOS}

Foi encontrada diferença significativa $(\mathrm{p}=0,002)$ entre os valores de produção de força isométrica máxima (em N) na condição BL e a soma dos valores unilaterais (ULD+ULE), estes últimos mostrando-se significativamente maiores (Figura 1).

Os valores de $\mathrm{IB}_{\mathrm{f}}$ mostraram-se significativamente menores do que zero $(\mathrm{p}<0,001)$, demonstrando presença de $\mathrm{DB}$ nos valores de produção de força isométrica máxima. Da mesma forma, foi encontrado DB na ativação muscular, uma vez que o IB ${ }_{\text {emg }}$ apresentou valores significativamente menores do que zero $(\mathrm{p}<0,001)$ (Tabela 1$)$.

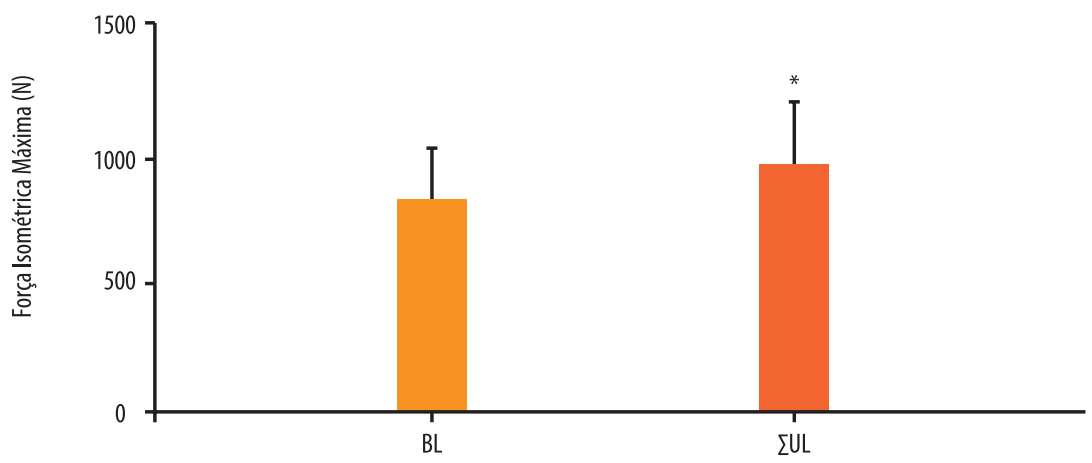

Figura 1. Média e desvio padrão da força isométrica máxima de extensão de joelhos correspondente à condição bilateral (BL) e ao somatório dos valores de cada membro inferior na condição unilateral ( $(\mathrm{UL})$. * diferença significativa entre as condições $(p=0,002)$.

Tabela 1. Média \pm desvio-padrão do índice bilateral (IB) da produção de força $\left(\mathrm{IB}_{\mathrm{f}}\right)$ e do sinal $\mathrm{EMG}\left(\mathrm{IB}_{\mathrm{emg}}\right)$.

\begin{tabular}{lc}
\hline Índice bilateral (IB) & Média \pm desvio-padrão \\
\hline $\mathrm{IB}_{\mathrm{f}}(\%)$ & $-15,7 \pm 10,0^{*}$ \\
$\mathrm{IB}_{\mathrm{emg}}(\%)$ & $-22,7 \pm 10,5 *$ \\
\hline
\end{tabular}

* Valores significativamente menores que zero = déficit bilateral (DB). 


\section{DISCUSSÃO}

Um dos principais achados no presente estudo foi que os valores relativos à produção de força e ao nível de ativação muscular (avaliada por EMG) obtidos na condição UL mostraram-se expressivamente superiores aos valores destas variáveis obtidos na condição $\mathrm{BL}$, resultados estes que corroboram dados reportados na literatura científica ${ }^{4-7}$. Embora não investigados no presente estudo, os mecanismos que parecem explicar esta diferença estão relacionados à ocorrência de inibição inter-hemisfério, na condição BL, bem como à maior ativação de fibras tipo II na condição UL. Durante a execução BL, em que há maior complexidade motora e ativação simultânea dos dois hemisférios cerebrais, parece haver menor ativação de cada hemisfério cerebral, o que causa uma redução significativa na ativação de unidades motoras (UM), e consequente menor produção de força nesta condição ${ }^{5}$. Essa inibição inter-hemisfério parece ser um dos fatores limitantes do desempenho muscular no modo de execução $\mathrm{BL}^{7,8}$. Já no modo UL, a capacidade máxima muscular, representada pela produção de força máxima e pela atividade elétrica muscular (sinal EMG), pode ser incrementada, uma vez que apenas um hemisfério cerebral está sendo usado neste modo de execução. Este fenômeno, em que são expressos menores valores de força produzidos no modo BL relativamente ao UL, representa o denominado déficit bilateral (DB).

O DB tem sido reportado nas contrações isométricas voluntárias máximas (CIVMs) em diferentes populações e exercícios ${ }^{4,5,9,10,11}$. Esse fenômeno pode ocorrer em pequenos e grandes grupos musculares, em exercícios mono ou multiarticulares, em ambos os sexos, bem como em atletas ou sedentários ${ }^{6,78,18}$. No entanto, parece não ser evidente em indivíduos que realizam no seu programa de treino exercícios em condição $\mathrm{BL}$, como é o caso de ciclistas, assim como em sujeitos que apresentam facilitação bilateral, como tem sido observado em levantadores de peso ${ }^{8}$.

A falta de consenso entre os resultados apresentados na literatura relativos à presença ou não do $\mathrm{DB}$ parece ser decorrente das diferentes metodologias utilizadas nos estudos. Protocolos em que contrações isométricas máximas são realizadas parecem propiciar um controle mais adequado de variáveis que podem influenciar os resultados, como o posicionamento e a estabilização corporal do indivíduo durante os testes, sobretudo, quando são avaliadas as condições neurais por EMG em ambas as condições (UL e BL ${ }^{19,20}$. Da mesma forma, protocolos que envolvem ações monoarticulares parecem ser mais adequados para avaliar as condições neurais associadas ao DB do que aqueles em que são utilizadas ações poliarticulares, nas quais há um número maior de músculos ativados (sinergistas) e maior variabilidade na ativação destes músculos durante o movimento. Embora em contrações isométricas haja um maior controle das variáveis acima referidas, existem variações entre as magnitudes de DB (relativo à força) reportadas na literatura. Howard e Enoka ${ }^{8}$ encontraram DB de aproximadamente $7 \%$ em condições isométricas, em destreinados, mas não o encontraram em levantadores de peso e ciclistas. Schantz et al. ${ }^{21}$, não encontraram DB e, ao contrário, registraram facilitação 
bilateral de $4 \%$ em sujeitos treinados e destreinados. Koh et al. ${ }^{10}$ encontraram em sujeitos destreinados DB de, aproximadamente, $17 \%$ a $24 \%$ em condições isométricas. Häkkinen et al. ${ }^{12}$ não o encontraram em contrações isométricas de extensores do joelho de homens e mulheres de meia idade e idosos. No estudo de Owings e Grabiner ${ }^{3}$, idosos apresentaram DB entre 6,5\% e 12,9\% em condições isométricas de extensão dos joelhos. Já no presente estudo foi observado um DB de aproximadamente 15\% na produção de força em sujeitos jovens.

Da mesma forma que os valores de força, no presente estudo, os valores de sinal EMG dos músculos RC e VL apresentaram-se maiores na condição UL ( $\sum$ sinal EMG ULD + ULE) em relação à condição $\mathrm{BL}(\Sigma$ sinal EMG BLD + BLE), reforçando que mais unidades motoras são ativadas na condição UL. Em conjunto, estes resultados mostram valores significativos de DB, calculado a partir do índice bilateral (IB), para os valores de sinal EMG, resultados estes que corroboram os resultados de outros estudos apresentados na literatura, em que também foi reportado o fenômeno ${ }^{4-7}$.

Owings e Grabiner ${ }^{3}$ sugerem que uma queda no sinal EMG em paralelo com uma menor produção de força, em condições bilaterais, implica uma relação de causa e efeito entre DB e fatores neurais, o que ficou claramente evidenciado no presente estudo, uma vez que foram encontrados menores valores tanto na produção de força como no sinal EMG na condição BL. O mesmo também tem sido observado em outros estudos desta natureza que compararam as condições de execução UL e BL em exercícios de extensão do joelho e flexão do cotovelo, isométrica e dinamicamente $e^{6,9,22}$.

A partir dos resultados do presente estudo e de questões levantadas na literatura científica ${ }^{8}$, é possível especular algumas aplicações práticas relativas às condições UL e BL: a) programas de treino de força compostos por exercícios executados na condição BL podem diminuir cronicamente $\mathrm{o}$ bloqueio do córtex cerebral, por aumentarem o recrutamento de unidades motoras, sobretudo, do tipo II, nesta condição, diminuindo, assim, o DB para a extensão de joelhos ${ }^{11,20,23}$; b) por outro lado, exercícios executados na condição UL tendem a aumentar cronicamente o DB, embora o nível de ativação muscular seja intensificado agudamente nesta condição ${ }^{11,20,23,}$; c) ainda que estudos tenham reportado que o treino UL incrementa o DB, o exercício de execução UL pode ser utilizado para casos de desequilíbrio muscular, bem como para atletas que necessitam dar mais importância à produção de força de um membro relativamente ao seu contralateral; d) exercícios unilaterais, por ativarem um menor volume muscular, parecem ser mais adequados para indivíduos hipertensos, pois promovem menores incrementos da pressão arterial do que exercícios bilaterais ${ }^{24}$. Ainda que estas especulações sejam pertinentes, os efeitos crônicos de exercícios de força realizados nas condições UL e BL necessitam ser mais investigados.

\section{CONCLUSÕES}

Os resultados do presente estudo ratificam a ocorrência do DB no exercício de extensão isométrica de joelhos, em sujeitos que não possuem prática 
do treinamento de força. Além disso, estes resultados reforçam a hipótese da limitação neural durante a condição de ação BL. Contudo, este estudo apenas revela o efeito agudo do exercício extensão de joelhos realizado nas condições UL e BL. Assim, seria interessante a realização de estudos longitudinais que avaliem o comportamento de variáveis neuromusculares e morfológicas em resposta aos treinos UL e BL, para que o fenômeno DB seja melhor compreendido em longo prazo, uma vez que não existe consenso na literatura quanto aos efeitos crônicos do treinamento realizado nestas condições.

\section{REFERÊNCIAS BIBLIOGRÁFICAS}

1. Fleck SJ, Kraemer WJ. Designing resistance training programs. Champaign: $\mathrm{Hu}-$ man Kinetics; 2004.

2. Kraemer WJ, Ratamess, NA. Fundamentals of resistance training: progression and exercise prescription. Med Sci Sports Exerc 2004;36(4):674-88.

3. Owings TM, Grabiner MD. Fatigue effects on the bilateral deficit are speed dependent. Med Sci Sports Exerc 1998;30(8):1257-62.

4. Owings TM, Grabiner MD. Normally aging older adults demonstrate the bilateral deficit during ramp and hold contractions. J Gerontol A Biol Sci Med Sci 1998;53A(6):B426-B429.

5. Van Dieën JH, Ogita F, De Haan A. Reduced neural drive in bilateral exertions: a performance-limiting factor? Med Sci Sports Exerc 2003;35:(1)111-8.

6. Kuruganti U, Murphy T. Bilateral deficit expressions and myoelectric signal activity during submaximal and maximal isometric knee extensions in young, athletic males. Eur J Appl Physio 2008;102(6):721-6.

7. Ohtsuki T. Decrease in human voluntary isometric arm strength induced by simultaneous bilateral exertion. Behav Brain Res 1983;7(2):165-78.

8. Howard JD, Enoka RM. Maximum bilateral contractions are modified by neurally mediated interlimb effects. J Appl Physiol 1991;70(1):306-16.

9. Vandervoort AA, Sale DG, Moroz J. Comparison of motor unit activation during unilateral and bilateral leg extension. J Appl Physiol 1984;56(1):46-51.

10. Koh TJ, Grabiner MD, Clough CA. Bilateral deficit is larger for step than for ramp isometric contractions. J Appl Physiol 1993;74(3):1200-5.

11. Kuruganti U, Parker P, Rickards J, Tingley M, Sexsmith J. Bilateral isokinetic training reduces the bilateral leg strength deficit for both old and young adults. Eur J Appl Physio 2005;94(1-2):175-9.

12. Hakkinen K, Kallinen M, Linnamo V, Pastinen UM, Newton RU, Kraemer WJ. Neuromuscular adaptations during bilateral versus unilateral strength training in middle-aged and elderly men and women. Acta Physiol Scand 1996;158(1):77-88.

13. Hakkinen K, Kraemer WJ, Newton RU. Muscle activation and force production during bilateral and unilateral concentric and isometric contractions of the knee extensors in men and women at different ages. Electromyogr Clin Neurophysiol 1997;37(3):131-42.

14. Jakobi JM, Cafarelli E. Neuromuscular drive and force production are not altered during bilateral contractions. J Appl Physiol 1998;84(1):200-6.

15. Herbert RD, Gandevia SC. Muscle activation in unilateral and bilateral efforts by motor nerve and cortical stimulation. J Appl Physiol 1996;80(4):1351-6.

16. Leis AA, Trapani VC. Atlas of Electromyography. Oxford: Oxford University Press; 2000.

17. Narici MV, Roi GS, Landoni L, Minetti AE, Cerretelli P. Changes in force, cross-sectional area and neural activation during strength training and detraining of human quadriceps. Eur J Appl Physiol 1989;59(4):310-9. 
18. Hay D, Souza AS, Fukashiro S. Human bilateral deficit during a dynamic multi-joint leg press movement. Hum Mov Sci 2006;25(2):181-91.

19. De Luca CJ. The use of electromyography in biomechanics. J Appl Biomec 1997;13(2):135-63.

20. Jakobi JM, Chilibeck PD. Bilateral and unilateral contractions: Possible differences in maximal voluntary force. Can J Appl Physiol 2001;26(1):12-33.

21. Schantz PG, Moritani T, Karlson E, Johansson E, Lundh A. Maximal voluntary force of bilateral and unilateral leg extension. Acta Physiol Scand 1989;136(2):185-92.

22. Oda S, Moritani T. Maximal isometric force and neural activity during bilateral and unilateral elbow flexion in humans. Eur J Appl Physiol 1994;69(3):240-3.

23. Taniguchi Y. Relationship between the modification of bilateral deficit in upper and lower limbs by resistance training in humans. Eur J Appl Physio 1998;78(3):226-30.

24. Cruz I, Rosa G, Santos EMR, Dias IBF, Simão R, Novaes J, et al. Acute answers of the blood pressure, heart beat and double product after the execution of the knees extension in a bilateral and unilateral way. Fit Perf J 2007;6(2):111-5.
Endereço para correspondência

Ronei Silveira Pinto.

LAPEX, Universidade Federal do Rio Grande do Sul, UFRGS.

Rua: Felizardo, 750.

Bairro: Jardim Botânico.

CEP: 90690-200 - Porto Alegre/ RS,

Brasil.

E-mail: ronei.pinto@ufrgs.br. 